\title{
BMJ Open Dynapaenic obesity and its association with health outcomes in older adult populations: protocol for a systematic review
}

\author{
Siobhan Leahy, ${ }^{\oplus 1}$ Marica Cassarino, ${ }^{2}$ Matthew DL O' Connell, ${ }^{3}$ Liam Glynn, ${ }^{4}$ \\ Rose Galvin ${ }^{5}$
}

To cite: Leahy S, Cassarino M, 0 ' Connell MDL, et al. Dynapaenic obesity and its association with health outcomes in older adult populations: protocol for a systematic review. BMJ Open 2019;9:e027728. doi:10.1136/ bmjopen-2018-027728

- Prepublication history and additional material for this paper are available online. To view please visit the journal (http:// dx.doi.org/10.1136/bmjopen2018-027728).

Received 5 November 2018 Revised 28 February 2019 Accepted 12 April 2019

Check for updates

(C) Author(s) (or their employer(s)) 2019. Re-use permitted under CC BY-NC. No commercial re-use. See rights and permissions. Published by BMJ.

For numbered affiliations see end of article.

Correspondence to Dr Siobhan Leahy; siobhan.leahy@ul.ie

\section{ABSTRACT}

Introduction Two major global health challenges are the rapidly ageing population and the high prevalence of obesity in all age groups. Older adults are also susceptible to age-related loss of muscle strength, termed dynapaenia. The co-occurrence of both obesity and dynapaenia, termed dynapaenic obesity (D0), has been associated with poorer health outcomes and increased healthcare usage compared with either state alone. The purpose of this systematic review is to quantify the prevalence and incidence of DO in older adult populations, and to explore the association between D0 and health outcomes, specifically chronic disease and multimorbidity, functional disability and healthcare usage.

Methods and analysis Using the Meta-analyses of Observational Studies in Epidemiology guidelines, we will conduct a systematic review of cross-sectional and longitudinal observational studies of older adults, which include measures of DO and specified outcomes. Detailed literature searches of will be conducted using six electronic databases: Excerpta Medica dataBASE (EMBASE), PubMed, MEDLINE, SCOPUS, ScienceDirect and Cumulative Index of Nursing and Allied Health Complete (CINAHL), including articles published from database inception until Febuary 2019. The reference lists of included articles will also be searched. Two independent reviewers will undertake a three-step screening and review process using the Population, Risk Factor, Outcome framework to define eligibility. The Newcastle Ottawa Scale for non-randomised studies will be used to assess risk of bias and to rate study quality. The findings will be synthesised in a narrative summary, and a meta-analysis will be conducted where appropriate.

Ethics and dissemination Ethical approval is not required for this systematic review. Findings from this research will be submitted for peer-reviewed publication in academic journals, and presented at relevant academic conferences.

PROSPERO registration number CRD42018112471.

\section{INTRODUCTION}

\section{Background}

Two major societal challenges at a global level are the rapidly ageing population and the
Strengths and limitations of this study

- This is the first systematic review to investigate the frequency of dynapaenic obesity (D0) and associated health outcomes in older adult populations.

- This review will benefit from a comprehensive search of six electronic health and biomedical databases using an extensive search strategy.

- We expect heterogeneity between studies in terms of both the criteria for classification of DO and the reporting of health .outcomes, which may lead to difficulties in conducting a meta-analysis.

- Results of this review will help to establish if DO is a significant risk factor for poor health outcomes in older adults, and may contribute to the design of future interventions to target those most at risk of this phenotype and its negative effects.

continual rise in obesity prevalence in people of all ages. ${ }^{12}$ The double burden of ageing and obesity therefore potentially represents a looming health sector crisis, whereby a greater number of older adults are living with multimorbidity ${ }^{3}$ and disability, much of which is related to modifiable risk factors such as obesity.

Evidence regarding the effect of obesity on morbidity and mortality in older populations is conflicting. While obesity is a recognised risk factor for chronic diseases such as cardiovascular disease, diabetes and some cancers, ${ }^{2}$ a number of studies have suggested that excess weight may be protective against adverse outcomes in many chronic conditions. This phenomenon is referred to as the 'obesity paradox' ${ }^{4}$ and is based on the use of body mass index (BMI) to measure obesity. BMI is an imperfect measure in older adults as weight stability, or even weight loss, may mask an increase in fat mass and the redistribution of fat to the abdominal area. ${ }^{5}$ This central or visceral accumulation of fat mass 
is more strongly associated with cardiometabolic disease than fat deposited at peripheral sites. ${ }^{6}$ Waist circumference (WC) measures, or a combination of WC and BMI, may therefore be a more useful measure of body composition in older adult populations.

In addition to changes in fat mass and its distribution, ageing is associated with a gradual decline in muscle mass, termed sarcopaenia and muscle strength, termed dynapaenia. ${ }^{7}$ Measures of sarcopaenia and dynapaenia are increasingly being investigated as predictors of functional decline and health outcomes in studies of older adults. ${ }^{8}$ However, as with the measurement of fat mass, it is difficult to directly measure sarcopaenia in large samples or routine clinical settings. Conversely, dynapaenia can be readily measured using grip dynamometry which is low cost, and commonly available in clinical settings. Dynapaenia is a component of the frailty syndrome, ${ }^{9}$ and obesity, measured by both BMI and WC has been associated with frailty in older adult populations. ${ }^{10}$ The co-occurrence of obesity and dynapaenia is termed 'dynapaenic obesity' (DO $)^{11}$; the co-occurrence of dynapaenia and central or abdominal obesity (measured using WC) is termed 'dynapaenic abdominal obesity' (DAO) ${ }^{12}$ While both conditions can occur independently as a natural part of the ageing process, it has been hypothesised that obesity and muscle impairment (in this case decreased muscle strength) may be pathophysiologically connected. Specific mechanisms through which obesity may exacerbate the process of dynapaenia include decreased physical activity, chronic low-grade inflammation, insulin resistance, hormonal changes and malnutrition, ${ }^{13}$ which may also predispose older adults to specific health impairments. A recent study on an older Italian cohort demonstrated an association between DAO and worsening disability and increased risk of hospitalisation, ${ }^{14}$ while Batsis $e t a l^{15}$ found DO to predict disability, functional decline and poorer quality of life in a study of US adults aged 45-79 years. Associations with type 2 diabetes prevalence ${ }^{16}$ and incidence, ${ }^{17}$ decreased bone mineral density ${ }^{18}$ and mortality ${ }^{12}$ have also been shown. The increased activity in this area of research in recent years necessitates the conduct of a systematic review and meta-analysis to determine (1) the frequency of DO in older adults and (2) the totality of evidence exploring a link between DO and health outcomes in this population.

\section{Objectives}

The purpose of this systematic review is to (1) quantify the prevalence and incidence of DO in older adult populations and (2) explore the association between DO and health outcomes, specifically (a) chronic disease and multimorbidity, (b) functional disability and (c) healthcare usage.

\section{METHODOLOGY}

This protocol has been prepared following the Preferred Reporting Items for Systematic review and Meta-Analysis
Protocols 2015 checklist $^{19}$ (online supplementary file 1 ), and has been registered with the international prospective register of systematic reviews (PROSPERO) database. Any protocol amendments will be declared in PROSPERO as they occur. The proposed systematic review and meta-analysis will be carried out according to Meta-analyses Of Observational Studies in Epidemiology guidelines. ${ }^{20}$

\section{Eligibility criteria}

We will include observational studies of older adult populations using either cross-sectional or longitudinal (prospective or retrospective) designs. The Population, Risk factor, Outcome framework has been employed to define eligibility criteria as outlined below.

\section{Population}

Our target study population is adults aged 50 years and older.

\section{Risk factor}

The risk factor or exposure of interest for this review is DO or DAO). Studies with objective measures of obesity (classified according to BMI and/or WC) and muscle strength (measured by hand grip or knee dynamometry) will be included.

\section{Outcome}

Prevalence and/or incidence of DO/DAO will be recorded as a percentage (\%) with $95 \%$ CI. Further outcomes of interest are the association between:

\section{DO/DAO and chronic disease}

Chronic disease will be defined as the presence of doctor diagnosed, long term health conditions. Several definitions of comorbidity and multimorbidity exist in the literature and all will be considered for inclusion in this review.

\section{DO/DAO and functional disability}

Studies which use a validated measure of disability, such as walking speed, the 36 -item short-form health survey, ${ }^{21}$ the Katz activities of daily living ability scale ${ }^{22}$ or Lawton Instrumental Activities of Daily Living, ${ }^{23}$ will be included in the review.

\section{DO/DAO and healthcare usage}

Specific indicators of healthcare usage will include general practitioner or family physician visits, outpatient and emergency department visits and hospital admissions.

\section{Search strategy}

A detailed literature search will be conducted in six electronic databases; Excerpta Medica dataBASE (EMBASE), PubMed, MEDLINE, SCOPUS, ScienceDirect and Cumulative Index of Nursing and Allied Health Complete. Grey literature including conference proceedings and institutional repositories will also be searched. The reference lists of included articles will be hand searched. Medical Subject Headings and synonyms relating to the study 
objectives will be used to target our search. Searches will be restricted to studies published in the English language using human subjects only. Articles published from database inception until February 2019 will be included in the review. A preliminary EMBASE search strategy has been developed in conjunction with the University of Limerick Faculty of Education and Health Sciences librarian and is detailed in online supplementary file 2. This strategy will be adapted and refined for each of the remaining databases.

\section{Data extraction}

A detailed record of all search strategies and results will be maintained to ensure reproducibility of the review. All results will be imported into the Rayyan citation management software, ${ }^{24}$ where duplicate citations will be screened and removed. A three stage reviewing process will be employed. Two reviewers (SL and RG) will screen all retrieved titles against the stated eligibility criteria. Following this, both reviewers (SL and RG) will independently screen all selected abstracts. SL and RG will then independently review the full text of included articles. A third reviewer (MC) will adjudicate on disagreements at all stages of the review process.

The following information will be extracted from included articles using a proforma template:

Study characteristics:

- Study design.

- Study location and setting.

- Sample size.

- Inclusion and exclusion criteria.

- Definition of risk factor/exposure, that is, whether DO or DAO was measured and what measurement tools were used.

- Definition of outcome measures.

Population characteristics:

- Age.

- Sex.

- Ethnicity.

- Prevalence/incidence of DO/DAO.

- Outcomes (association with disease/disability/healthcare usage).

\section{Assessing study quality}

The Newcastle Ottawa Scale for quality assessment of non-randomised studies ${ }^{25}$ will be used independently by two reviewers (SL and RG) to assess risk of bias and to rate study quality. Disagreements will be adjudicated by a third reviewer (MC).

\section{Data synthesis}

Initial analysis will extract data on prevalence and/or incidence of DO/DAO from the included studies. Studies will then be grouped according to the three outcomes of interest: (1) association with chronic disease burden, (2) association with functional disability and (3) association with indicators of healthcare usage. These groupings may not be mutually exclusive.
A narrative synthesis will be conducted to summarise the characteristics of the included studies. If possible, a random effects meta-analysis of included studies will be carried out in consultation with a statistician. Outcomes will be recorded as crude or adjusted risk ratio or crude or adjusted OR with 95\% CI, depending on the study type. Variables used to derive adjusted estimates will be recorded where relevant. In cases where CIs are not provided, variance estimates will be derived from $p$ values as per standard methods. ${ }^{26}$ Data will be pooled using Review Manager V.5.2, developed by the Cochrane Collaboration. ${ }^{27}$ Forest plots will be generated and the pooled estimates of effect will be suppressed as required. Publication bias (eg, Kendall's tau) and heterogeneity (eg, the $\mathrm{I}^{2}$ statistic) will also be examined. Subgroup analysis by sex, age group (eg, 50-64 years, 65 years+), DO definition (DO, DAO) and study design will be performed where possible. Finally, a sensitivity check will be conducted to determine the consistency and robustness of the findings based on the methodological quality of the included studies.

The Grading of Recommendations, Assessment, Development and Evaluation guideline will be used to assess the strength of the body of evidence, as recommended by the Cochrane Handbook. ${ }^{28}$

\section{Ethics and dissemination}

This review constitutes secondary analysis of existing data and does not require ethical approval. The findings of this research will be submitted for peer-reviewed publication in academic journals, and presented at relevant seminars and conferences and through traditional and social media channels.

\section{Patient and public involvement}

Patients and public will not be involved in this study. The research questions addressed in this review arose from clinical observations of older adults. We anticipate that the findings of this review (which represents Phase I of the Medical Research Council framework for developing and evaluating complex interventions ${ }^{29}$ ) will aid in the design of a pilot intervention to address the older population at risk of DO and associated adverse health outcomes. These subsequent phases will have a strong patient and public involvement (PPI) component.

\section{DISCUSSION/CONCLUSION}

This systematic review will synthesise existing evidence to quantify the prevalence and incidence of DO and associated health outcomes in older adult populations. This will help to establish if $\mathrm{DO} / \mathrm{DAO}$ is a significant risk factor for poor health outcomes in older adults, and may contribute to the design of future primary care interventions to target those most at risk of this phenotype and its negative effects.

There are a number of potential strengths and limitations to the planned review that will be considered when 
discussing the findings. Heterogeneity between studies, in terms of methodologies and outcome measures used, may limit our ability to conduct a meta-analysis or to draw reliable conclusions from the available evidence. Restricting included articles to the English language is also a potential limitation. The use of observational studies is a strength of our review as there is likely to be large sample sizes more likely to be representative of the overall population, aiding generalisability of the results. Our adherence to established guidelines for the conduct of systematic reviews and critical appraisal will also ensure the quality of the final review.

To the best of our knowledge, no reviews have been previously published exploring this research question. Should a relevant review be published during the preparation of our review, it will be incorporated into our review and meta-analysis if feasible.

\section{Author affiliations}

${ }^{1}$ Faculty of Education and Health Sciences, Health Research Institute, University of Limerick, Limerick, Ireland

${ }^{2}$ School of Allied Health, University of Limerick Faculty of Education and Health Sciences, Limerick, Ireland

${ }^{3}$ Warwick Medical School, University of Warwick, Coventry, West Midlands, UK ${ }^{4}$ Graduate Entry Medical School and Health Research Institute, University of Limerick, Limerick, Ireland

${ }^{5}$ School of Allied Health, Faculty of Education and Health Sciences, Health Research Institute, University of Limerick, Limerick, Ireland

Contributors SL and RG were major contributors to drafting the manuscript. $\mathrm{SL}, \mathrm{RG}$ and $\mathrm{LG}$ designed the study. SL developed the search strategy. MC and MDLOC participated in the project design and critically appraised and edited the manuscript. SL is the guarantor of the review. All authors read and approved the final manuscript. The corresponding author attests that all listed authors meet authorship criteria and that no others meeting the criteria have been omitted.

Funding The authors have not declared a specific grant for this research from any funding agency in the public, commercial or not-for-profit sectors.

Competing interests None declared.

Patient consent for publication Not required.

Provenance and peer review Not commissioned; externally peer reviewed.

Open access This is an open access article distributed in accordance with the Creative Commons Attribution Non Commercial (CC BY-NC 4.0) license, which permits others to distribute, remix, adapt, build upon this work non-commercially, and license their derivative works on different terms, provided the original work is properly cited, appropriate credit is given, any changes made indicated, and the use is non-commercial. See: http://creativecommons.org/licenses/by-nc/4.0/.

\section{REFERENCES}

1. World Health Organisation. World report on ageing and health. Geneva: World Health Organisation, 2015.

2. World Health Organisation. Global Health Observatory (GHO) data Overweight and obesity. Geneva: World Health Organisation, 2017.

3. Glynn LG, Valderas JM, Healy P, et al. The prevalence of multimorbidity in primary care and its effect on health care utilization and cost. Fam Pract 2011;28:516-23.

4. Hainer V, Aldhoon-Hainerová I. Obesity paradox does exist. Diabetes Care 2013;36(Suppl 2):S276-S281.
5. Villareal DT, Apovian CM, Kushner RF, et al. Obesity in older adults: technical review and position statement of the American Society for Nutrition and NAASO, The Obesity Society. Obes Res 2005;13:1849-63.

6. Klein S, Allison DB, Heymsfield SB, et al. Waist Circumference and Cardiometabolic Risk. A Consensus Statement from Shaping America's Health: Association for Weight Management and Obesity Prevention; NAASO, The Obesity Society; the American Society for Nutrition; and the American Diabetes Association. Obesity 2007:30:1647-52.

7. Clark BC, Manini TM. Sarcopenia != Dynapenia. J Gerontol A Biol Sci Med Sci 2008;63:829-34.

8. Manini TM, Clark BC. Dynapenia and aging: an update. J Gerontol A Biol Sci Med Sci 2012;67:28-40.

9. Fried LP, Tangen CM, Walston J, et al. Frailty in older adults: evidence for a phenotype. J Gerontol A Biol Sci Med Sci 2001;56:M146-M157.

10. Hubbard RE, Lang IA, Llewellyn DJ, et al. Frailty, body mass index, and abdominal obesity in older people. J Gerontol A Biol Sci Med Sci 2010;65:377-81.

11. Bouchard DR, Janssen I. Dynapenic-obesity and physical function in older adults. J Gerontol A Biol Sci Med Sci 2010;65:71-7.

12. Rossi AP, Fantin F, Caliari C, et al. Dynapenic abdominal obesity as predictor of mortality and disability worsening in older adults: A 10year prospective study. Clin Nutr 2016;35:199-204.

13. Stenholm S, Harris TB, Rantanen T, et al. Sarcopenic obesity: definition, cause and consequences. Curr Opin Clin Nutr Metab Care 2008:11:693-700.

14. Rossi AP, Bianchi L, Volpato S, et al. Dynapenic Abdominal Obesity as a Predictor of Worsening Disability, Hospitalization, and Mortality in Older Adults: Results From the InCHIANTI Study. J Gerontol A Biol Sci Med Sci 2017;72:1098-104.

15. Batsis JA, Zbehlik AJ, Pidgeon D, et al. Dynapenic obesity and the effect on long-term physical function and quality of life: data from the osteoarthritis initiative. BMC Geriatr 2015;15:118.

16. Kawakami R, Sawada SS, Lee IM, et al. Dynapenic Obesity and Prevalence of Type 2 Diabetes in Middle-Aged Japanese Men. $J$ Epidemiol 2015;25:656-62.

17. Cuthbertson DJ, Bell JA, Ng SY, et al. Dynapenic obesity and the risk of incident Type 2 diabetes: the English Longitudinal Study of Ageing. Diabet Med 2016;33:1052-9.

18. Scott $D$, Chandrasekara SD, Laslett LL, et al. Associations of Sarcopenic Obesity and Dynapenic Obesity with Bone Mineral Density and Incident Fractures Over 5-10 Years in CommunityDwelling Older Adults. Calcif Tissue Int 2016:99:30-42.

19. Moher D, Shamseer L, Clarke M, et al. Preferred reporting items for systematic review and meta-analysis protocols (PRISMA-P) 2015 statement. Syst Rev 2015;4:1.

20. Stroup DF, Berlin JA, Morton SC, et al. Meta-analysis of observational studies in epidemiology: a proposal for reporting. Meta-analysis Of Observational Studies in Epidemiology (MOOSE) group. JAMA 2000;283:2008-12.

21. Ware JE, Sherbourne CD. The MOS 36-item short-form health survey (SF-36). I. Conceptual framework and item selection. Med Care 1992;30:473-83.

22. Wallace M, Shelkey M. Reliability and Validity of Katz ADL Index. AJN, American Journal of Nursing 2008;108:1.

23. Lawton MP. The functional assessment of elderly people. J Am Geriatr Soc 1971;19:465-81.

24. Ouzzani M, Hammady H, Fedorowicz Z, et al. Rayyan-a web and mobile app for systematic reviews. Syst Rev 2016:5:210.

25. Wells GA, Shea B, O'Connell D, et al. The Newcastle-Ottawa Scale (NOS) for assessing the quality of nonrandomised studies in metaanalyses. http://www.ohri.ca/programs/clinical_epidemiology/oxford. asp (Accessed 7 Feb 2019).

26. Greenland S, Robins JM. Estimation of a common effect parameter from sparse follow-up data. Biometrics 1985;41:55-68.

27. The Cochrane Collaboration. Review Manager (RevMan) [Computer program]. Copenhagen: The Nordic Cochrane Centre, The Cochrane Collaboration, 2014.

28. Balshem $\mathrm{H}$, Helfand $\mathrm{M}$, Schünemann $\mathrm{HJ}$, et al. GRADE guidelines: 3 . Rating the quality of evidence. J Clin Epidemiol 2011;64:401-6.

29. Craig P, Dieppe P, Macintyre S, et al. Developing and evaluating complex interventions: the new Medical Research Council guidance. BMJ 2008;337:a1655. 